\title{
Evaluation de la digestibilité des drêches fraíches de brasserie chez le poney
}

\author{
R. WOLTER, J.P. VALETTE et Agnès DASTE \\ avec la collaboration technique de Mauricette Tournoux \\ Ecole nationale vétérinaire d'Alfort, Laboratoire de Nutrition-Alimentation \\ 7, avenue du Général-de-Gaulle, F 94704 Maisons-Allort
}

\begin{abstract}
Résumé
Six poneys placés en cage à métabolisme reçoivent successivement trois régimes à base de drêches fraîches $(0,65,6$ et 80,6 p. 100 de la matière sèche alimentaire) et d'un aliment "complet», à raison de 35,30 et $20 \mathrm{~g} \mathrm{MS} / \mathrm{kg} \mathrm{PV", \pi n).}$

Il en ressort que les drêches de brasserie sont un aliment de bonne valeur nutritive (CUD de matière organique $=66$ p. 100), en particulier grâce à la haute digestibilité des matières azotées $(84 \mathrm{p} .100)$ et des matières grasses ( 82 p. 100).

Néanmoins, leur utilisation, sous forme frâ̂che, ne peut sc concevoir qu'à proximité de brasseries, à caluse de leur instabilité.
\end{abstract}

\section{Introduction}

Les drêches de brasseries, obtenues après filtration, lors du brassage du malt de l'orge, constituent le résidu le plus important de l'industrie de la bière (PICcioni, 1965 ; Lubret, 1978 ; Daste, 1982) et entrent depuis longtemps dans l'alimentation du cheval (Lavalard, 1912).

La production française, estimée à 400000 tonnes/an est exportéc pour moitié vers les pays de la C.E.E. Parmi ceux-ci, les Pays-Bas en utilisent 450000 tonnes comme matière première pour les aliments complets (Louvard, 1980 ; LEBras \& PaNNET, 1981).

Les problèmes de conservation des drêches fraîches, la concentration géographique des brasseries dans la région Nord et en Alsace, ainsi que le coût énergétique de la déshydratation, conduisent à une utilisation des drêches sous forme d'ensilage.

Différentes études ont été menées sur la digestibilité et la valeur nutritive des drêches fraîches ou ensilées chez les ruminants (BALZER et al., 1974 ; CoTTYN et al., 1975 ; Demarquilly, 1979 ; Sauvant et al., 1982). De même, des essais de production chez des vaches laitières (BALzer et al., 1971 ; Broster et al., 1975 ; Chase, 1977 ; Parrassin, Verite \& Hoden, 1982) ont montré la bonne valeur alimentaire des drêches de brasserie.

L'intérêt zootechnique de ce sous-produit de brasserie étant prouvé chez les polygastriques, il nous a semblé intéressant, faisant suite à une série de travaux sur l'utilisation digestive de diverses matières premières (WOLTER et al., 1979-1980), d'évaluer, chez le poney, la digestibilité des drêches fraîches, associées à un aliment complet du commerce. 


\section{Matériel et méthodes}

\section{A. Animaux}

Six poneys mâles, adultes, de plus de 10 ans, dont les poids varient de 155 à $220 \mathrm{~kg}$, sont placés dans des cages à digestibilité munies de mangeoires amovibles, et d'abreuvoirs automatiques ne permettant pas de contrôler la consommation d'eau. Les animaux, dont les poids ont peu varié au cours de l'expérimentation, prennent de l'exercice pendant les périodes d'adaptation (15 jours). Ils restent entravés pendant les périodes de récolte ( 5 jours).

\section{B. Aliments et rations}

En phase expérimentale, l'ingestion maximale de drêches fraîches (1) a atteint $8 \mathrm{~kg} /$ jour, en 4 repas. Par la suite, elle a progressivement baissé, bien que l'approvisionnement soit renouvelé 2 fois par semaine et que les drêches soient stockées à $4{ }^{\circ} \mathrm{C}$ avant d'être distribuées aux animaux. En conséquence, nous avons dû associer une part d'aliment composé «complet» (2), et un complément minéral vitaminé (CMV) ${ }^{(3)}$, afin de maintenir une consommation suffisante et de prévenir d'éventuelles carences.

\section{TABLEAU 1}

Nature des régimes expérimentaux.

Experimental diets.

\begin{tabular}{|c|c|c|c|}
\hline $\begin{array}{c}\text { Nature des régimes } \\
\text { Diets }\end{array}$ & $\begin{array}{l}\text { En } \% \\
\text { de la ration } \\
\% \text { of diet }\end{array}$ & $\begin{array}{c}\text { En \% } \\
\text { de matière sèche } \\
\text { alimentaire } \\
\% \text { of dry matter }\end{array}$ & $\begin{array}{c}\text { Quantité ingérée } \\
\mathrm{g} \mathrm{MS} / \mathrm{kg} \mathrm{PV} \mathrm{PV}^{0,75} \\
\text { Intake } \\
\text { g DM/kg LW } W^{0.75}\end{array}$ \\
\hline $\begin{array}{l}\text { 1. Aliment complet } \ldots \ldots \ldots \text {. } \\
\text { Commercial feed }\end{array}$ & 100 & 100 & 35 \\
\hline $\begin{array}{l}\text { 2. Drêches ............. } \\
\text { Wet brewers grains }\end{array}$ & 88 & 65,6 & \\
\hline $\begin{array}{l}\text { Aliment complet } \\
\text { Commercial feed }\end{array}$ & 11 & 31,3 & 30 \\
\hline $\begin{array}{l}\text { CMV } \ldots \ldots \ldots \ldots \ldots \ldots \\
\text { Mineral and vitamin suppl }\end{array}$ & 1 & 3,1 & \\
\hline $\begin{array}{l}\text { 3. Drêches } \ldots \ldots \ldots \ldots \ldots \\
\text { Wet brewers grains }\end{array}$ & 94,1 & 80,6 & \\
\hline $\begin{array}{l}\text { Aliment complet } \ldots \ldots \ldots \\
\text { Commercial feed }\end{array}$ & 4,7 & 15,5 & 20 \\
\hline $\begin{array}{l}\text { CMV } \ldots \ldots \ldots \ldots \ldots \ldots \\
\text { Mineral and vitamin suppl }\end{array}$ & 1,2 & 3,9 & \\
\hline
\end{tabular}

(1) Grâcieusement fournies par la brasserie « 33 Export» à Drancy. Kindly supplied by the brewery «33 Export» at Drancy.

(2) «Cheval Manège» Sanders (à base d'avoine, d'orge, de caroubes, de son fin, de tourteau de soja et de luzerne déshydratée).

(Based on oats, barley, carob, fine bran, soyabean and dehydrated lucerne.)

(3) «Agglobov » Sanders. 
Les régimes expérimentaux retenus figurent au tableau 1 .

Les compositions centésimales des aliments et des régimes sont rapportées au tableau 2.

\section{Tableau 2}

Composition des matières premières et des régimes étudiés (\%).

Composition of raw materials and diets (\%).

\begin{tabular}{|c|c|c|c|c|c|}
\hline & $\begin{array}{c}\text { Drêches } \\
\text { Wet } \\
\text { brewer's } \\
\text { grains }\end{array}$ & $\begin{array}{c}\text { Aliment } \\
\text { complet } \\
=\text { régime } \mathbf{I} \\
\text { Diet } I\end{array}$ & $\mathrm{CMV}^{*}$ & $\begin{array}{c}\text { Régime II } \\
\text { (drêches : } \\
65,6 \% \text { ) } \\
\text { Diet II }\end{array}$ & $\begin{array}{l}\text { Régime IH } \\
\text { (drêches : } \\
80,6 \% \text { ) } \\
\text { Diet III }\end{array}$ \\
\hline $\begin{array}{l}\text { Matière sèche } \ldots \ldots \ldots \ldots \\
\text { Dry matter }\end{array}$ & 23,4 & 89,2 & 89,1 & 31,4 & 27,3 \\
\hline $\begin{array}{c}\text { En } \% \text { de } \mathrm{MS} \\
\% \text { of } D M\end{array}$ & & & & & \\
\hline $\begin{array}{l}\text { MAT }(\mathrm{N} \times 6,25) \\
\text { Crude protein }\end{array}$ & 27,0 & 16,7 & 2,4 & 23,0 & 24,4 \\
\hline $\begin{array}{l}\text { Cellulose brute } \ldots \ldots \ldots \\
\text { Crude cellulose }\end{array}$ & 18,3 & 18,1 & - & 17,7 & 17,6 \\
\hline $\begin{array}{l}\text { NDF } \ldots \ldots \ldots \ldots \ldots \ldots \\
\text { Total fibre }\end{array}$ & 64,2 & 35,9 & - & 53,3 & 57,3 \\
\hline $\begin{array}{l}\mathrm{ADF} \\
\text { Lignocellulose }\end{array}$ & 22,4 & 20,7 & - & $2 \cdot 1,2$ & 21,3 \\
\hline $\begin{array}{l}\text { Hémicelluloses } \ldots \ldots \\
\text { (NDF - ADF) }\end{array}$ & 41,8 & 15,2 & - & 32,1 & 36,0 \\
\hline $\begin{array}{l}\text { Lignine } \ldots \ldots \ldots \ldots \\
\text { Lignin }\end{array}$ & 7,8 & 7,7 & - & 7,5 & 7,5 \\
\hline $\begin{array}{l}\text { Cellulose « vraie } » . . . . \\
\text { True cellulose }\end{array}$ & 14,6 & 13,0 & - & 13,7 & 13,8 \\
\hline $\begin{array}{l}\text { Matières grasses } \ldots \ldots \ldots \\
\text { Fat }\end{array}$ & 8,3 & 3,7 & - & 6,6 & 7,2 \\
\hline $\begin{array}{l}\text { Matières minérales } \\
\text { Ash }\end{array}$ & 3,8 & 8,7 & 65,8 & 7,3 & 7,0 \\
\hline $\begin{array}{l}\text { Matière organique } \ldots \ldots \\
\text { Organic matter }\end{array}$ & 96,2 & 91,3 & 34,2 & 92,7 & 93,0 \\
\hline
\end{tabular}

(*) Complément minéral vitaminé - Mineral and vitamine supplement. 


\section{Méthodes d'analyses}

Les digestibilités sont déterminées après récolte totale des fèces pendant 5 jours.

Les prélèvements quotidiens de 20 p. 100 des matières fécales totales sont aspergés avec une solution de $\mathrm{H}_{2} \mathrm{SO}_{4}$ à 2 p. 100 afin de limiter les pertes d'azote volatil ; ils sont ensuite homogénéisés ; une partie est séchée à $80^{\circ} \mathrm{C}$ pendant 72 heures, puis broyée et conservée en chambre froide jusqu'au moment des analyses.

Les échantillons, aliments et fèces, sont soumis aux analyses suivantes :

- M.A.T. $(\mathrm{N} \times 6,25)$, par la méthode de Kjeldahl ;

- cellulose brute, par la technique de Weende;

- constituants pariétaux, selon le procédé de VAN SoEsr (1963);

- matières grasses, par extraction à l'éther de pétrole ;

— matières minérales, après incinération à 550 " $\mathrm{C}$ pendant 5 heures.

Les résultats expérimentaux sont soumis aux tests suivants selon SchwarTz (1969) : le test « $t$ » de Student par la méthode des couples, l'étude de la corrélation, de la régression et de la linéarité, l'analyse de variance.

\section{Résultats et discussion}

Les résultats de lanalyse des drêches (tabl. 2). font ressortir que le total NDF + MAT + MG + cendres dépasse 100 p. 100 . Ceci s'explique sans doute par le fait qu'une part de l'azote est comptabilisée 2 fois. En effet, 9,5 p. 100 des MAT $(\mathrm{N} \times 6,25)$, soit 35 p. 100 des protéines totales, se trouvent dosés avec les glucides pariétaux, très vraisemblablement à la suite de la réaction de Maillard qui accompagne le maltage. En conséquence, les taux de NDF et ADF, voire de lignine, sont surévalués; ceci peut d'ailleurs contribuer à rendre compte d'une digestibilité relativement élevée de ces composants membranaires, d'autant plus que la proportion de drêches augmente dans le régime.

Les coefficients d'utilisation digestive (moyennes et intervalles de confiance $\mathrm{m} \pm \mathrm{t} \mathrm{s} / \sqrt{\mathrm{n}}$, $\mathrm{s}$ étant l'écart-type estimé) des différents régimes sont récapitulés dans le tableau 3 .

- La digestibilité apparente des protéines est excellente (82, 83,9 et 82,9 p. 100), en rapport avec les teneurs élevées en azote de chacun des régimes.

- De même, la digestibilité des matières grasses est relativement bonne et s'améliore $(74,79,3$ et 81,2$)$ avec l'augmentation du taux lipidique du régime.

- La digestibilité moyenne de la cellulose se situe vers 40 à 50 p. 100 mais subit d'assez larges variations individuelles.

- La digestibilité de l'ADF montre une certaine similitude avec celle de la cellulose brute. 


\section{TABleau 3}

Coefficients d'utilisation digestive des régimes (\%).

Digestibilities of diets (\%).

\begin{tabular}{|c|c|c|c|}
\hline & $\begin{array}{c}\text { Régime I } \\
\text { (drêches : } 0 \% \text { ) } \\
\text { Diet I } \\
(0 \% \text { brewers grains) }\end{array}$ & $\begin{array}{c}\text { Régime II } \\
\text { (drêches : } 65,6 \% \text { ) } \\
\text { Diet II } \\
\text { (65.6\% brewers } \\
\text { grains) }\end{array}$ & $\begin{array}{c}\text { Régime III } \\
\text { (drêches : } 80,6 \% \text { ) } \\
\text { Diet } \text { III } \\
\text { (80.6\% brewers } \\
\text { grains) }\end{array}$ \\
\hline $\begin{array}{l}\text { Matière sèche } \cdots \cdots \\
\text { Dry matter }\end{array}$ & $67,8^{a} \pm 4,4$ & $64,2^{b} \pm 4,2$ & $61,7^{b} \pm 5,0$ \\
\hline $\begin{array}{l}\text { MAT } \ldots \ldots \ldots \ldots \ldots \\
\text { Crude protein }\end{array}$ & $82,0 \mathrm{a} \pm 2,9$ & $83,9^{a} \pm 2,2$ & $82,9^{a} \pm 1,8$ \\
\hline $\begin{array}{l}\text { Matière organique } \ldots \\
\text { Organic matter }\end{array}$ & $71,2^{a} \pm 4,0$ & $67,6^{\prime \prime} \pm 3,7$ & $66,5^{b} \pm 5,5$ \\
\hline $\begin{array}{l}\text { Matières grasses } . . . \\
\text { Fat }\end{array}$ & $74,0^{a} \pm 4,4$ & $79,3^{\mathrm{b}} \pm 5,0$ & $81,2^{\mathrm{b}} \pm 3,7$ \\
\hline $\begin{array}{l}\text { Ceilulose brute } \\
\text { Crude cellulose }\end{array}$ & $39,2^{a} \pm 9,1$ & $44,4^{a} \pm 8,3$ & $51,0^{\mathfrak{a}} \pm 5,9$ \\
\hline $\begin{array}{l}\text { NDF } \ldots \ldots \ldots \cdots \\
\text { Total fibre }\end{array}$ & $42,6^{a} \pm 9,4$ & $62,2^{\mathrm{b}} \pm 4,7$ & $63,4^{b} \pm 7,4$ \\
\hline $\begin{array}{l}\text { ADF ................. } \\
\text { Lignocellulose }\end{array}$ & $33,0^{\mathrm{a}} \pm 12,8$ & $49,6^{b} \pm 6,2$ & $54,6^{b} \pm 4,6$ \\
\hline $\begin{array}{l}\text { Hémicelluloses } \ldots \ldots \\
(\mathrm{NDF}-\mathrm{ADF})\end{array}$ & $56,1^{a} \pm 4,8$ & $71,5^{\mathrm{b}} \pm 3,9$ & $70,5^{b} \pm 8,4$ \\
\hline$\underset{\text { Lignin }}{\text { Lignine }} \ldots \ldots \ldots \ldots \ldots$ & $18,1^{\mathrm{a}} \pm 13,1$ & $49,7^{b} \pm 8,6$ & $63,9 \mathrm{~b} \pm 2,9$ \\
\hline $\begin{array}{l}\text { Cellulose «vraie» } \\
\text { True cellulose }\end{array}$ & $41,7 \mathfrak{a} \pm 12,9$ & $49,7^{b} \pm 6,5$ & $50,0^{\mathrm{b}} \pm 5,5$ \\
\hline
\end{tabular}

a, b : Moyennes significativement différentes au seuil 5 p. 100.

Means significantly different at the level of 5 p. 100.

L'analyse de variance entre le régime témoin 1 et les deux traitements (régimes 2 et 3) montre que ces deux derniers sont identiques et diffèrent du régime 1 , exceptions faites pour les digestibilités des MAT et de la cellulose. La méthode des couples, test plus performant, entre les régimes 1 et 2 , confirme ces données, mais fait apparaître en plus une différence significative concernant la digestibilité de la cellulose.

Nous pouvons donc affirmer que l'incorporation de drêches fraîches de brasserie à cet aliment composé complet, détériore quelque peu la digestibilité de la matière sèche et de la matière organique de l'ensemble du régime et donc sa valeur nutritive. 
- Le but essentiel de ce travail étant de déterminer la digestibilité des drêches, impossible en consommation exclusive à cause de la relative inappétibilité de cette matière première; nous y sommes parvenus par deux moyens :

- le premier à partir des droites de régression de la digestibilité en fonction du taux d'aliment composé complet dans la ration $(15,5,31,3$ et 100$)$, après avoir vérifié la linéarité des droites ainsi obtenues. On obtient :

(a) CUD régime $=$ pente $\times A+$ CUD drêches

avec $\mathrm{A}=$ pourcentage d'aliment complet dans la ration;

- le second, par substitution, grâce à la relation : $\mathrm{CMV} \times \mathrm{C}$

(b) CUD régime $=$ CUD aliment complet $\times \mathrm{A}+$ CUD drêches $\times \mathrm{B}+$ CUD

avec $\mathbf{A}, \mathbf{B}, \mathbf{C}=$ proportion de l'élément considéré en provenance de l'aliment complet, des drêches, du CMV, dans la ration.

Par simplification, on admet une digestibilité totale du CMV mais toute erreur à ce propos semble négligeable compte tenu des très faibles taux d'incorporation du CMV (C).

Le tableau 4 indique les coefficients de digestibilité des drêches obtenus par la droite de régression (a) pour :

$A=0$, et par substitution (b) dans les régimes II et III, ainsi que les coefficients de corrélation (il n'y a pas de corrélation pour MAT et cellulose «vraie», car les pentes des droites de régression ne sont pas différentes de 0 ).

Les deux estimations donnent des résultats comparables et si la méthode par substitution est moins rigoureuse, elle a l'avantage de n'exiger qu'un seul régime expérimental.

Dans l'ensemble, les drêches apparaissent comme un aliment de bonne valeur énergétique comme en témoigne la digestibilité de la matière organique qui les situe au niveau du maïs fourrage et de la luzerne déshydratée (Wolter et al., 1979).

L'utilisation digestive de la matière azotée est remarquable et s'explique par la très forte teneur en MAT des drêches. La digestibilité des fractions cellulosiques est comparable à celle de la paille sodée (Wolter, Valette \& Morel-GAray, 1982) ou des céréales comme l'avoine ou l'orge (WoLTER et al., 1982).

A la suite de Fahey, McLaren \& Williams (1979), nous confirmons que la lignine est un mauvais marqueur pour l'évaluation de la digestibilité (CUD $=68,4$ p. 100). Il semblerait que des complexes du chrome ou du cobalt avec l'EDTA soient plus appropriés aux mesures de digestibilité chez les équidés (UDEN, Collucci, VAN SoEst, 1980).

La très bonne digestibilité des matières grasses $(82-83$ p. 100), vérifie la relativement haute tolérance des équidés à ces constituants alimentaires.

Dans l'ensemble, nos résultats sont en accord avec ceux obtenus par CotTYN et al. (1975) sur des béliers, ainsi que par SAUVANT et al. (1982) chez les ruminants laitiers. 


\section{Tableau 4}

Estimation de la digestibilité des drêches (\%).

Estimated digestibility of wet brewer's grains (\%).

\begin{tabular}{|c|c|c|c|c|}
\hline & $\begin{array}{l}\text { Coefficient de corrélation } \\
\text { seuil } 5 \%=0,50 \\
\text { Coefficient of correlation } \\
\text { Level of significance } \\
5 \%=0.50\end{array}$ & Régression (a) & Substit & tution (b) \\
\hline $\begin{array}{l}\text { Matière sèche } \ldots \ldots \ldots \\
\text { Dry matter }\end{array}$ & 0,54 & 61,6 & 59 & -61 \\
\hline $\begin{array}{l}\text { MAT } \ldots \ldots \ldots \ldots \\
\text { Crude protein }\end{array}$ & $\begin{array}{c}0,31 \\
\text { ns }\end{array}$ & 83,9 & 83 & $-84,5$ \\
\hline $\begin{array}{l}\text { Matière organique } \ldots \ldots \ldots \\
\text { Organic matter }\end{array}$ & 0,52 & 65,8 & 65 & $-65,5$ \\
\hline $\begin{array}{l}\text { Matières grasses } \ldots \ldots \ldots \\
F A T\end{array}$ & 0,63 & 82,1 & 81 & -84 \\
\hline $\begin{array}{l}\text { Cellulose brute } \ldots \ldots \ldots \\
\text { Crude cellulose }\end{array}$ & 0,51 & 50,2 & 47 & -53 \\
\hline $\begin{array}{l}\text { NDF } \ldots \\
\text { Total fibre }\end{array}$ & 0,85 & 69,1 & 65,5 & -67 \\
\hline $\begin{array}{l}\text { ADF } \ldots \ldots \ldots \ldots \ldots \ldots \\
\text { Lignocellulose }\end{array}$ & 0,77 & 57,9 & 55,5 & -58 \\
\hline $\begin{array}{l}\text { Hémicelluloses } \ldots \ldots \\
\text { (NDF - ADF) }\end{array}$ & 0,86 & 75,7 & 71,5 & -74 \\
\hline $\begin{array}{l}\text { Lignine } \\
\text { Lignin }\end{array}$ & 0,91 & 68,4 & 63 & -71 \\
\hline $\begin{array}{l}\text { Cellulose « vraie } » \ldots \ldots \ldots \\
\text { True cellulose }\end{array}$ & $\begin{array}{c}0,44 \\
\text { ns }\end{array}$ & 52,4 & 51 & $-52,5$ \\
\hline
\end{tabular}

\section{Conclusion}

Les drêches de brasserie constituent un aliment de bonne valeur nutritive chez les équidés, comme chez les autres espèces domestiques.

Toutefois, leur utilisation comme source azotée chez les équidés se trouve plus limitée que chez les ruminants, en raison de leur médiocre valeur biologique.

La forme fraîche, surtout, restreint fortement les possibilités d'approvisionnement, de conservation et de stockage, étant donné la localisation des brasseries, le volume et l'instabilité de cette matière première. 
En outre, en France, le prix de revient actuel des drêches n'est intéressant qu'à proximité d'une brasserie par suite de l'inorganisation d'un réseau de distribution.

Néanmoins, certains pays, comme la Hollande, ont compris l'avantage à tirer parti de ce produit de substitution dans l'alimentation animale.

L'efficacité zootechnique des drêches est prouvée même chez les équidés, mais reste à savoir si économiquement, leur utilisation est rentable en France.

Cette étude gagnerait à être complétée par une expérience similaire avec des drêches de brasserie ensilées, plus aisées d'emploi, ou mieux encore avec des drêches sèches, sans doute plus appétentes.

Accepté pour publication en juillet 1983.

\section{Summary}

Digestibility of wet brewer's grains in ponies

Six ponies placed in metabolism crates were fed successively three diets based on brewer's grains $(0,65.6$ and 80.6 p. 100 of the dry matter $)$ and a commercial feed $(35,30$ and $20 \mathrm{~g} \mathrm{DM} / \mathrm{kg} \mathrm{LW}^{0.75}$ ).

Brewers wet grains were found to have a good nutritive value (apparent digestibility coefficient $=66$ p. 100), particularly because of the high digestibility of both crude protein $(84$ p. 100$)$ and fat $(82$ p. 100$)$.

Nevertheless, because of their instability, they can only be used as wet grain in the neighbourhood of breweries.

\section{Références bibliographiques}

Balzer G., Feger R., Morhain B., Vignon B., 1974. Les drêches de brasseries dans l'alimentation de bovins à viande. Bull. Tech. Inf., 294, 827-836.

Balzer G., Barbezant H., Feger R., Spindler F., 1971. Un aliment protéique mal utilisé en France : les drêches de brasserie. Bull. Tech. Inf., 261, 603-622.

Broster W.H., Broster V.J., Smith T., Sniter J.W., 1975. Experiment on the nutrition of the dairy heifer. IX - Food utilization in lactation. J. Agric. Sci., 84, 173-186.

ChaSe L.E., 1977. Brewer's grain, an old feed with a new future. Proc. Cornell Nutr. Conf., 99-103.

Cottyn B.G., Boucque C.V., Aerts J.V., Buysse F.X., 1975. Digestibilité et valeur alimentaire des drêches de brasserie frâiches ou ensilées. Rev. Agric., 28, 1519-1525.

Daste A., 1982. Evaluation de la digestibilité des drêches fraîches de brasserie chez le poney. Rapport de stage. $3^{\mathrm{e}}$ année de zootechnie. Institut National Agronomique, Paris, $109 \mathrm{p}$.

Demarquilly C., 1979. Valeur nutritive et utilisation des drêches de brasserie. Bull. Tech. Inf., 343-344, 389-393.

Fahey G.C., McLaren G.A., Williams J.E., 1979. Lignin digestibility by lambs fed both low quality and high quality roughages. J. Anim. Sci., 48, 941-946.

Lavalard E., 1912. L'alimentation du cheval. Librairie agricole de la Maison Rustique de Paris, 164 p. 
Legras B., Pannet Y., 1981. Utilisation et commercialisation des drêches de brasserie aux Pays-Bas. Prod. Lait. Mod., 94, 13-16.

Louvard G., 1980. Les vaches hollandaises mangent de plus en plus de drêches françaises. Elev. Bov., 96, 35-43.

Lubret J.M., 1978. Les drêches de brasserie et leur utilisation dans l'alimentation des bovins. Thèse pour le Doctorat Vétérinaire, Toulouse, $45 \mathrm{p}$.

Parrassin P.R., Verite R., Hoden A., 1982. Utilisation des drêches de brasseries ensilées par les vaches laitières. Bull. Tech. C.R.Z.V. Theix, I.N.R.A., 47, 27-32.

Piccioni M., 1965. Dictionnaire des aliments pour les animaux. Ed. Agricole, Bologne, 640 pages.

Sauvant D., Giger S., Dorleans M., Hervieu J., Diallo I., 1982. Contributions à l'étude de la valeur alimentaire des drêches de brasserie pour le ruminant laitier (à paraître).

Schwartz D., 1969. Méthodes statistiques à l'usage des médecins et des biologistes. Flammarion Médecine Sciences. Paris, 318 p.

Uden P., Colucci P.E., van Soest P.S., 1980. Investigations of chromium, cerium and cobalt as markers as digesta. Rate of passage studies. J. Sci. Food Agric., 31, 625-632.

VAN SoEST P.S., 1963. Use of detergent in the analysis of fibrous feed. II - A rapid method for the determination of fibre and lignin. J. Assoc. Off. An. Chem., 46, 829-835.

Wolter R., Durix A., Letourneau J.C., Carcelen M., 1979. Evaluation chez le poney de la digestibilité du maïs-fourrage déshydraté, des pulpes sèches de betterave, de la luzerne déshydratée, du son de blé, de la paille de blé et des pulpes de raisins. Ann. Zootech., 28, 93-100.

Wolter R., Durix A., Letourneau J.C., Carcelen M., 1980. Evaluation chez le poney de la digestibilité des pellicules de soja, du marc de pommes, des caroubes et du tourteau de pépins de raisins. Ann. Zootech., 29, 377-385.

Wolter R., Valette J.P., Durix A., Letourneau J.C., Carcelen M., 1982. Digestibilité comparée de quatre céréales (avoine, orge, maïs, blé) selon le mode de présentation, chez le poney. Ann. Zootech., 31, 445-458.

Wolter R., Valette J.P., Morel-Garay G., 1982. Digestibilité de la paille traitée à la soude chez le poney. Ann. Zootech., 31, 459-470. 